\title{
Article \\ Improvement in Strain Sensor Stability by Adapting the Metal Contact Layer
}

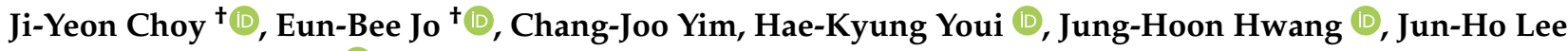 \\ and Hyun-Seok Kim * (1)
}

Citation: Choy, J.-Y.; Jo, E.-B.; Yim, C.-J.; Youi, H.-K.; Hwang, J.-H.; Lee, J.-H.; Kim, H.-S. Improvement in Strain Sensor Stability by Adapting the Metal Contact Layer. Sensors 2022, 22, 630. https://doi.org/10.3390/ s22020630

Academic Editors: Jung Woo Sohn and Heung Soo Kim

Received: 18 December 2021

Accepted: 11 January 2022

Published: 14 January 2022

Publisher's Note: MDPI stays neutral with regard to jurisdictional claims in published maps and institutional affiliations.

Copyright: (C) 2022 by the authors. Licensee MDPI, Basel, Switzerland. This article is an open access article distributed under the terms and conditions of the Creative Commons Attribution (CC BY) license (https:// creativecommons.org/licenses/by/ $4.0 /)$.

\author{
Division of Electronics and Electrical Engineering, Dongguk University, Seoul 04620, Korea; \\ jiyeon9711@naver.com (J.-Y.C.); eunbee.j123@gmail.com (E.-B.J.); akreal@naver.com (C.-J.Y.); \\ yeahwooong@gmail.com (H.-K.Y.); junghoonh0326@gmail.com (J.-H.H.); junho_1211@naver.com (J.-H.L.) \\ * Correspondence: hyunseokk@dongguk.edu; Tel.: +82-2-2260-3996 \\ + These authors contributed equally to this work.
}

\begin{abstract}
Research on stretchable strain sensors is actively conducted due to increasing interest in wearable devices. However, typical studies have focused on improving the elasticity of the electrode. Therefore, methods of directly connecting wire or attaching conductive tape to materials to detect deformation have been used to evaluate the performance of strain sensors. Polyaniline (PANI), a ptype semiconductive polymer, has been widely used for stretchable electrodes. However, conventional procedures have limitations in determining an appropriate metal for ohmic contact with PANI. Materials that are generally used for connection with PANI form an undesirable metal-semiconductor junction and have significant contact resistance. Hence, they degrade sensor performance. This study secured ohmic contact by adapting Au thin film as the metal contact layer (the MCL), with lower contact resistance and a larger work function than PANI. Additionally, we presented a buffer layer using hard polydimethylsiloxane (PDMS) and structured it into a dumbbell shape to protect the metal from deformation. As a result, we enhanced steadiness and repeatability up to $50 \%$ strain by comparing the gauge factors and the relative resistance changes. Consequently, adapting structural methods (the MCL and the dumbbell shape) to a device can result in strain sensors with promising stability, as well as high stretchability.
\end{abstract}

Keywords: stretchable sensor; strain sensor; wearable device; polyaniline; PDMS

\section{Introduction}

Highly flexible and stretchable next-generation wearable devices are anticipated for applications in various fields, such as flexible robotics, displays, smart watches for healthcare, and functional clothing. Accordingly, it is appropriate to conduct in-depth research aimed at developing a high-sensitivity strain sensor that can operate with stability despite deformation, while in contact with human skin, in light of the increasing demand for body-mounted devices.

Studies on strain sensors that can convert mechanical strain, such as body movement, into electrical signals have been actively pursued [1]. Novel electrodes and substitutes for typical metal or inorganic films have been studied to ensure conductivity and stretchability under strain, including metal nanowires [2], conductive polymers [3,4], the carbon nanotube (CNT) [5], graphene [6], and composites of these devices.

In particular, polyaniline (PANI), one of the conductive polymers, has attracted attention as an appropriate substance, due to its environmental stability, cost-effectiveness, and tunable conductivity [7], which provides scalability in a multi-functional strain sensor capable of sensing various stimuli, such as chemical gases, temperatures, and humidity [8]. However, strain sensors made by spin-coating or printing PANI dispersed in a solvent are limited because it is difficult to secure high elasticity due to their rigid chain structure. 
Cai et al. recently demonstrated stretchability of up to $150 \%$ with high sensitivity, and excellent mechanical adaptability via the island-bridge morphology of PANI film using dilute-chemical polymerization, resulting in the expansion of PANI's versatility as a stretchable electrode [9].

Studies on stretchable electrodes have been actively conducted. However, studies on packaging, integration, and measurement of strain sensors have not been sufficiently conducted [10]. Silver paste for wire connections, conductive tapes such as copper tape, and conductive fabric have been directly attached to stretchable electrodes for estimating their performance [11]. However, such methods have several problems. First, it is difficult to detect minute strain because the deformed region of the stretchable electrode cannot be precisely controlled. Second, contact resistance, based on the unstable attachment and increased instability under external stress, degrades a device's efficiency. Moreover, the effectiveness of materials that have been used for measurement, including copper and silver, are limited.

There is a restriction in establishing a stable metal semiconductor junction with the various stretchable electrodes. In particular, PANI is a p-type semiconductor whose conductivity results from delocalization by the $\pi$-conjugation structure, filling the local electrondeficient part of the cation radical. Thus, its work function is dependent on synthetic methods, such as chemical, electrochemical, and interfacial methods, synthetic conditions, such as the conditions related to dopants, acids, and solvents, and the ambient environment.

The typical work function of PANI is in the range of $4.2-4.7 \mathrm{eV}$. It should have ohmic contact with metal within this range to achieve high sensitivity [12-14]. However, it was not possible to form ohmic contact or to reduce contact resistance by simply employing a metal tape. An additional step, such as using silver paste, has been required [7,9]. In addition, most devices do not come with a separate contact area or appropriate connecting methods [15-17]. Therefore, adapting a metal contact layer to sensors could assure ohmic contact.

Silicone-based elastomers, such as polydimethylsiloxane (PDMS), Ecoflex, Dragon Skin, and hydrogel, are commonly used as stretchable substrates for body-attached devices. In particular, PDMS is frequently used due to its high elasticity, chemical stability, biological compatibility, transparency, and thermal stability [18]. Additionally, adjusting the mixing ratio of its components and curing conditions can easily control physical properties $[19,20]$. However, most studies on PDMS have focused on surface treatment, molding, or adhesion improvement. Hence, fabrication methods for a single substrate that is composed of more than two types of PDMS with different physical rigidities, using their advantages, have not been suggested.

Therefore, only a substrate with a single PDMS is provided for stretchable sensors or their applications. Hence, when a metal thin film that is susceptible to deformation is applied to sensors configured with PDMS, polymers such as polyimide and parylene have been required as a protective layer [21]. Furthermore, although studies on the fabrication of metal patterning on PDMS have advanced, their low surface energy hinders the application of photoresist, due to non-uniformity and low adhesion, preventing precise pattern formation. Additionally, its coefficient of thermal expansion, swelling, and flexibility acts as a hindrance $[22,23]$. Consequently, the patterning on PDMS via conventional photolithography is difficult and restricted.

This paper introduces a study that ultimately aims to improve the operational stability of strain sensors by adapting the metal contact layer (the MCL). We fabricated a strain sensor that included PANI as a sensing material to detect deformation, the MCL to retain ohmic contact with PANI, and a buffer layer to prevent breakage of the MCL by external stress. Additionally, we cut the sensor in the shape of a dumbbell to maximize the protective effect of the MCL and the buffer layer. Furthermore, the possibility of patterning PANI, the MCL, and the buffer layer is suggested, to provide potential for a diverse selection of materials on stretchable electrodes or the MCL ( $\mathrm{Au}$ and Ag), indicating the versatility of fabrication of stretchable strain sensors. 
The buffer layer formation was established from the facile controllability of PDMS's physical stiffness. We fabricated a single substrate composed of two different types of PDMS. As a result, it has high elasticity, but partial regions which have low elasticity can be protected from deformation. These partial regions, the buffer layers, can prevent breakage or peel-off of the MCL during the fabrication or application. The buffer layer is PDMS with a Young's modulus greater than $3 \mathrm{MPa}$ (more than $6 \mathrm{MPa}$ in this study). It is called hard PDMS (H-PDMS) because it is larger than the general Young's modulus. The substrate that determines the overall elasticity of the sensor has a Young's modulus less than $1 \mathrm{MPa}$ (less than $700 \mathrm{kPa}$ in this study); it is called soft PDMS (S-PDMS) because its modulus is smaller than the general Young's modulus. Furthermore, we carried out the PDMS patterning process to formulate PDMS with different physical properties within a single substrate. We introduced the transfer with a sacrificial layer and an auxiliary substrate to minimize damage during the process.

PANI, the MCL, and the H-PDMS can be formed on a single plane with a facile procedure. We demonstrated promising stability, reproducibility, and classification of novel structured sensors based on a comparison of the gauge factors (GFs) of three samples, depending on the MCL and the buffer layer and their transient measurement of relative resistance change under repeated tensile stress. Moreover, current-voltage (I-V) graphs of three types of sensors with different positions of the MCL according to PANI lengths were compared to identify the effect of the dumbbell-shape that was designed in accordance with the standards of the American Society for Testing of Materials (ASTM) [24-26].

\section{Materials and Methods}

\subsection{Materials}

Aniline (>99.5\%, ACS reagent, 62-53-3), sulfuric acid (98\%, for analysis EMSURE, 766493-9), hydrochloric acid (37\%, ACE reagent, 7647-01-0), (3-Aminoprophyl) triethoxysilane (99\%, 2554-06-05), and 2,4,6,8-tetramethyl-2,4,6,8-tetravinylcyclotetrasiloxane (2554-0605) were purchased from Sigma-Aldrich, Seoul, Korea. A graphite rod (GR002H) was purchased from QRINS, Seoul, Korea. ITO-coated glass was purchased from iTASCO, Seoul, Korea. A silicone elastomer base and a curing agent (Sylgard 184 kit) were purchased from Dow Corning, Midland, MI, USA. VDT-731 (67762-94-1), SIT-6831.2 (68478-92-2), and HMS-301 (68037-59-2) were purchased from Gelest, Morrisville, PA, USA. Copper tape was purchased from $3 \mathrm{M}$, Seoul, Korea. All reagents were used as received without further purification.

\subsection{Characterization}

The thickness of PDMS and PANI was analyzed using a surface profiler (Alpha Step 200, KLA/TENCOR). The alignment of PANI, Au thin film (the MCL), and the HPDMS were observed using a microscope (MX51, Olympus, Tokyo, Japan). The surface morphology of transferred PANI was analyzed via FE-SEM (S-4800, Hitachi, Tokyo, Japan) at the Korea Advanced Nano Fab Center.

\subsection{Fabrication of Strain Sensor}

\subsubsection{PANI/Au/H-PDMS Strain Sensor}

We introduced a strain sensor with the MCL and the buffer layer (PANI/Au/H-PDMS strain sensor). Its fabrication process is shown in Figure 1. First, we prepared the ITO-coated glass. It was cleaned with acetone, IPA, and deionized water prior to processing. Patterning directly on PDMS may cause alignment difficulties. A transfer process using ITO (185 nm, $10 \Omega / \mathrm{cm}^{2}$ ) as a sacrificial layer and glass as an auxiliary substrate was used (Figure 1a). ITO was chosen as a sacrificial layer because it can act as a working electrode during subsequent PANI synthesis. Typical photolithography was performed to establish the MCL. Photoresist (AZ5214E, AZ Electronics Materials, Charlotte, NC, USA) was spin-coated at $3000 \mathrm{rpm}$ for $30 \mathrm{~s}$. After the exposure, it was immersed in developer (CD-30, AZ Electronics Materials, Charlotte, NC, USA) for $1 \mathrm{~min} 10 \mathrm{~s}$. Au, which has a significantly larger work function 
than PANI, was selected as the MCL to form a stable ohmic contact. Thereafter, $150 \mathrm{~nm}$ of $\mathrm{Au}$ thin film was deposited via an e-beam evaporator (KVE-T5560, Korea Vacuum Tech, Gimpo, Korea), using Au pellets (purity 99.999\%) as a source. The deposition conditions were as follows: $2.8 \times 10^{-5}$ Torr, $140 \mathrm{~mA}$, and an $0.8 \mathrm{k} \AA / \mathrm{s}$ of deposition rate. It was lifted into a rectangular shape $(3000 \mu \mathrm{m} \times 1010 \mu \mathrm{m})$ and served as the MCL (Figure $1 \mathrm{~b})$.

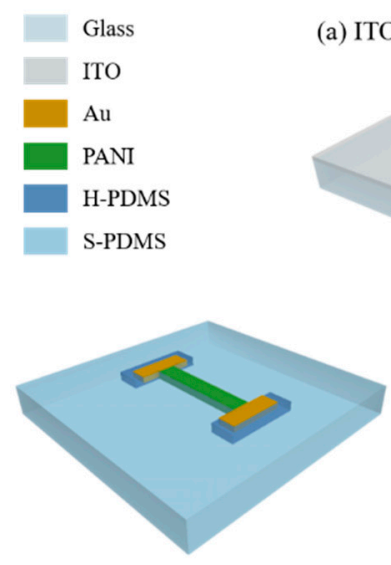

(g) Flip PDMS and cut (a) ITO-coated glass

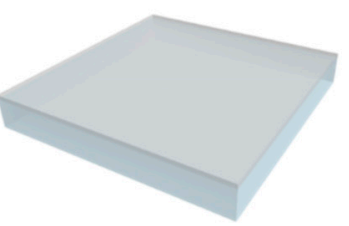

(b) Deposit Au and lift off

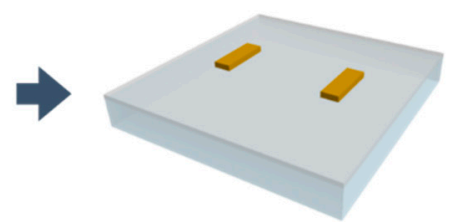

(c) Synthesize PANI pattern

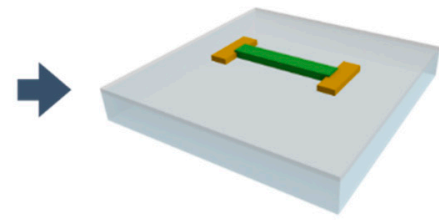

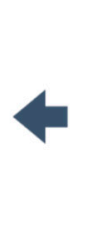

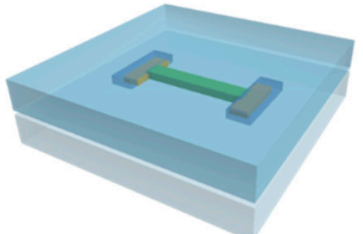

(f) Etch ITO
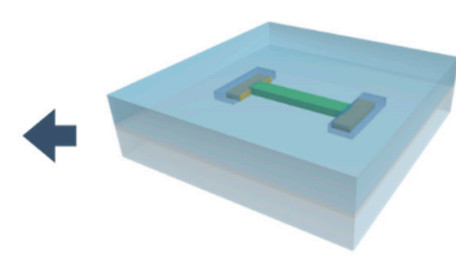

(e) Spin S-PDMS and cure

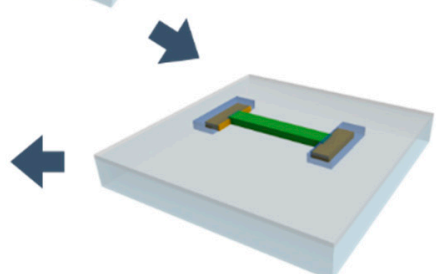

(d) Patterning H-PDMS

Figure 1. Fabrication of PANI/Au/H-PDMS strain sensor: (a) preparing the auxiliary substrate with a sacrificial layer; (b) formation of MCL; (c) formation of sensing material; (d) formulation of the buffer layer; (e) formulation of the stretchable substrate; (f) separating the stretchable substrate and the auxiliary substrate; (g) cutting PDMS into a dumbbell shape.

Next, photolithography was implemented to define PANI capable of detecting mechanical strain. A PANI mask was aligned to overlap with $20 \mu \mathrm{m}$ of the MCL. A thicker photoresist (KPRO-15, KemLab, Woburn, MA, USA) was used to ensure PANI thickness and spin-coated at $1000 \mathrm{rpm}$ for $45 \mathrm{~s}$. PANI was synthesized electrochemically. Photoresist acted as a deposition mask for patterning (width of $1500 \mu \mathrm{m}$ and length of $8270 \mu \mathrm{m}$ ). The polymerization conditions were as follows: $0.3 \mathrm{M}$ of aniline and $0.7 \mathrm{M}$ aqueous solution of sulfuric acid as an electrolyte, with the ITO-coated glass as a working electrode and the graphite rod as a counter electrode. The electrodeposition was performed for $1 \mathrm{~min} 10 \mathrm{~s}$ at a fixed voltage of $1.1 \mathrm{~V}$ at room temperature [7]. Thereafter, it was baked on the hot plate at $100{ }^{\circ} \mathrm{C}$ for $10 \mathrm{~min}$ to enhance adhesion to the substrate and dried at room temperature for $24 \mathrm{~h}$ to completely remove moisture. It was then soaked in acetone for $10 \mathrm{~min}$ to remove the photoresist and the residue was cleaned with IPA and deionized water (Figure 1c).

Subsequently, the H-PDMS patterning was conducted, as described in detail in Section 2.4 (Figure 1d). The S-PDMS determines the stretchability of a strain sensor. It was manufactured by mixing the base and a curing agent (Sylgard 184) with a weight ratio of 15:1. Prior to spin-coating the mixture, the curing agent was spin-coated at $3000 \mathrm{rpm}$ for $30 \mathrm{~s}$ to ensure adhesion to the H-PDMS. The mixture was then spin-coated at $500 \mathrm{rpm}$ for $30 \mathrm{~s}$ and sufficiently cured at room temperature for $72 \mathrm{~h}$ (Figure 1e). Finally, the ITO film was removed by the etchant, which is a diluent of $\mathrm{HCl}(37 \%)$, and deionized water with a weight ratio of 1:3 (Figure 1f). In this step, partial PANI that lost conductivity during the process was oxidized to emeraldine salt form and restored in an acidic etchant having low $\mathrm{pH}$. The PDMS film separated from the glass as the ITO was etched, flipped, and washed with deionized water. After that, it was cut into a dumbbell shape based on ASTM D412 (Figure 1g) [24-26]. Additionally, three samples with different PANI lengths $(5020 \mu \mathrm{m}, 7020 \mu \mathrm{m}$, and $8270 \mu \mathrm{m})$ were fabricated under the same conditions to optimize the strain sensor according to the position of the MCL and the buffer layer within the same dumbbell structure. 


\subsubsection{PANI and PANI/Au Strain Sensor}

We compared the strain sensor consisting of only PANI (PANI strain sensor) and the PANI/Au/H-PDMS strain sensor to demonstrate the effectiveness of adapting MCL. Additionally, a strain sensor without the buffer layer (PANI/ Au strain sensor) and PANI/Au/HPDMS strain sensor were compared to verify the efficiency of the buffer layer under deformation. The conditions of fabricating PANI, the MCL, and the S-PDMS in these samples were the same as those for the PANI/Au/H-PDMS strain sensors. In the case of the PANI strain sensor, the S-PDMS was applied immediately after defining the PANI on the ITO-coated glass. The sacrificial layer was then etched. PDMS film separated from the glass was cleaned with deionized water and cut into a rectangular shape $(7 \mathrm{~mm} \times 30 \mathrm{~mm})$. The dumbbell shape was not required because it was composed only of PANI, excluding the MCL and the buffer layer that require structural protection.

The PANI/Au strain sensor was fabricated by coating the S-PDMS on the patterned MCL and PANI. Similarly, the MCL and PANI were aligned with $20 \mu \mathrm{m}$ of the overlapping area. The surface treatment with (3-Aminoprophyl) triethoxysilane (APTES) was processed to enhance the adhesion between the S-PDMS and the MCL just before the S-PDMS coating. An aqueous solution diluted APTES with a weight ratio of 1:100 was used. It was dried with an $\mathrm{N}_{2}$ gun without a further washing process after immersion of $20 \mathrm{~min}$ [27]. After that, the S-PDMS was coated and cured for a sufficient time. The sacrificial layer was removed using $\mathrm{HCl}$ diluent to isolate it from the glass. It was washed by deionized water and cut into the dumbbell shape to protect the MCL structurally.

\subsection{Patterning the H-PDMS}

We applied the H-PDMS with a high Young's modulus as the buffer layer to protect the MCL from deformation. We introduced its patterning process as shown in Figure 2. First, the thick photoresist was applied to the sample to complete the step shown in Figure 1c, to ensure sufficient thickness that is fundamental for a durable buffer layer. We spin-coated the photoresist (KPRO-15, KemLab, Woburn, MA, USA) at $1000 \mathrm{rpm}$ for $45 \mathrm{~s}$ and soft-baked it to form the first photoresist layer. After cooling, a sufficient thickness of $35 \sim 40 \mu \mathrm{m}$ was obtained by repeating the same process under identical conditions. Thereafter, a pattern with a size of $4000 \mu \mathrm{m} \times 1500 \mu \mathrm{m}$ was aligned with PANI overlapped $10 \mu \mathrm{m}$ and the bulk MCL via conventional photolithography (Figure 2a). To develop the photoresist to a thickness greater than $35 \mu \mathrm{m}$, it was immersed in developer (CD-30, AZ Electronics Materials, Charlotte, NC, USA) for 20 min. APTES surface treatment was performed prior to applying the H-PDMS to improve adhesion between the MCL and the H-PDMS. After immersing in APTES aqueous solution diluted at a weight ratio of 1:100 for $20 \mathrm{~min}$, it was taken out and dried with an $\mathrm{N}_{2}$ gun.

(a) Pattern photoresist

(b) Spin H-PDMS and cure

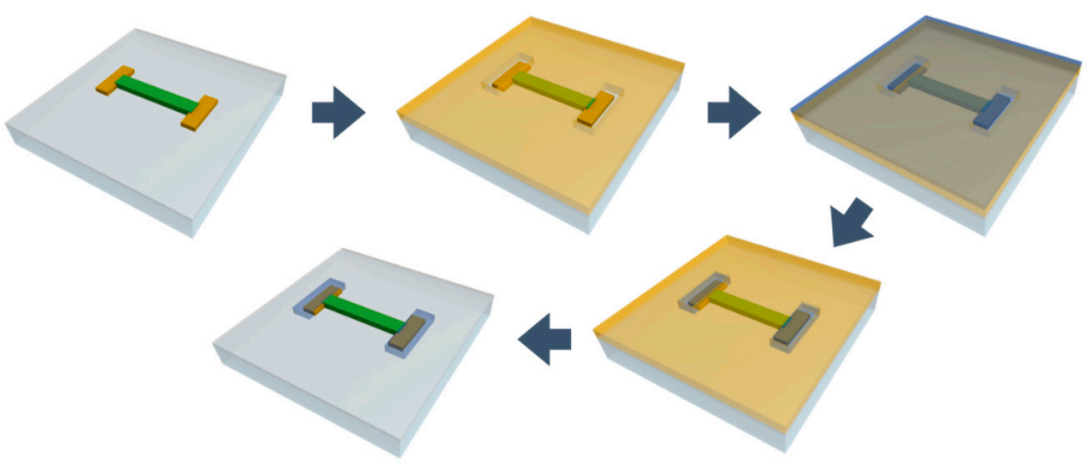

(d) Remove photoresist

(c) ICP-RIE

Figure 2. The process of patterning the H-PDMS: (a) photoresist as an etching mask; (b) formation of H-PDMS; (c) etching the top of H-PDMS to expose photoresist surface; (d) remained H-PDMS as the buffer layer. 
The H-PDMS was established in the following steps. First, $3.7 \mathrm{~g}$ of VDT-731 and $50 \mu \mathrm{L}$ of Pt catalyst (SIP-6831.2) were mixed. Then, $0.1 \mathrm{~g}$ of modulator (2,4,6,8-tetramethyl-2,4,6,8tetravinylcyclotetrasiloxane) and $1 \mathrm{~g}$ of HMS-301 were added and mixed. The mixture was degassed whenever each substance was added [28,29]. The mixture was spin-coated at $1000 \mathrm{rpm}$ for $30 \mathrm{~s}$. Self-leveling for $10 \mathrm{~min}$ on a flat place at room temperature was performed immediately after the preceding step. It was then cured in an oven at $60{ }^{\circ} \mathrm{C}$ for $4 \mathrm{~h}$ (Figure 2b). ICP-RIE was performed to expose the top surface of the photoresist by etching the H-PDMS. The selectivity of the H-PDMS photoresist was 1.5:1. The following were the ICP-RIE conditions: $500 \mathrm{~W}$ of RF power, $200 \mathrm{~W}$ of RF bias power, 10 mTorr of pressure with $90 \mathrm{sccm}$ of $\mathrm{SF}_{6}$ and $6 \mathrm{sccm}$ of $\mathrm{O}_{2}$, which lasted $32 \mathrm{~min}$ (Figure 2c) [30]. It was then immersed in an acetone solution to remove the photoresist. Notably, the exposed top surface facilitated its removal. The thickness of the remaining H-PDMS was $12 \mu \mathrm{m}$. The H-PDMS containing $10 \mu \mathrm{m}$ of PANI and the bulk MCL and an overlapped area with $10 \mu \mathrm{m}$ of the MCL and bulk PANI, situated externally to the H-PDMS, guaranteed the interconnection of each part under strain. Additionally, we manufactured Ag/H-PDMS samples and observed the variation of the surface conditions and the resistance based on strain to verify the effectiveness of the H-PDMS. Ag $(150 \mathrm{~nm})$ as the MCL and the H-PDMS $(25 \mu \mathrm{m})$ as the buffer layer were progressed under the same conditions as mentioned above. The S-PDMS was also formed identically using the mixture of a Sylgard 184 kit with a 15:1 weight ratio, and cut into the dumbbell shape.

\subsection{Measurement Conditions}

We prepared three different types of samples with and without the Au thin film as the MCL and the H-PDMS as the buffer layer: Sample A (PANI strain sensor), Sample B (PANI/Au strain sensor), and Sample C (PANI/Au/H-PDMS strain sensor). Sample A was cut into a rectangular shape because it lacked metallic thin film and was vulnerable to external stress. Samples B and C were cut into the shape of the dumbbell based on the ASTM D412 model to maximize the efficiency of the MCL protection. Each sample had a PANI pattern, $1500 \mu \mathrm{m}$ of width and $8270 \mu \mathrm{m}$ of length, which can detect mechanical deformation. We also prepared three different lengths (5020, 7020, and $8270 \mu \mathrm{m})$ of PANI in Sample $C$ to explore the effect of the dumbbell structure. The copper tape was prepared in size $0.125 \mathrm{~cm} \times 3 \mathrm{~cm}$ and attached directly to PANI or the MCL to measure the electrical properties (Figure 3). The measurement was repeated consecutively for all samples under the same conditions to obtain $\mathrm{I}-\mathrm{V}$ characteristics. Measurement conditions were as follows: dual sweep from $-10 \mathrm{~V}$ to $10 \mathrm{~V}$ with $0.1 \mathrm{~V}$ step voltage in a fixed state with deformation at $0 \%, 10 \%, 20 \%, 30 \%, 40 \%$, and 50\%. Transient characteristics were measured at speeds of $50 \mathrm{~mm} / \mathrm{min}$ and $6 \mathrm{~mm} / \mathrm{min}$. The former had a waiting time of $10 \mathrm{~s}$, and the latter had a waiting time of $0.1 \mathrm{~s}$. Ag/H-PDMS samples were cut into the ASTM D412 model, and their surface condition was observed using a microscope at $0 \%, 20 \%$, and $60 \%$ strains. Resistance was measured under the $0 \%, 20 \%, 40 \%$, and $60 \%$ strains using the Au probe. 


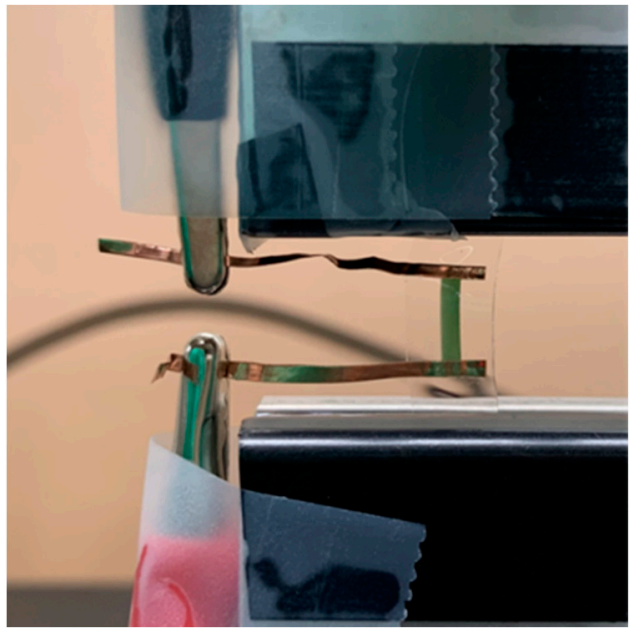

(a)

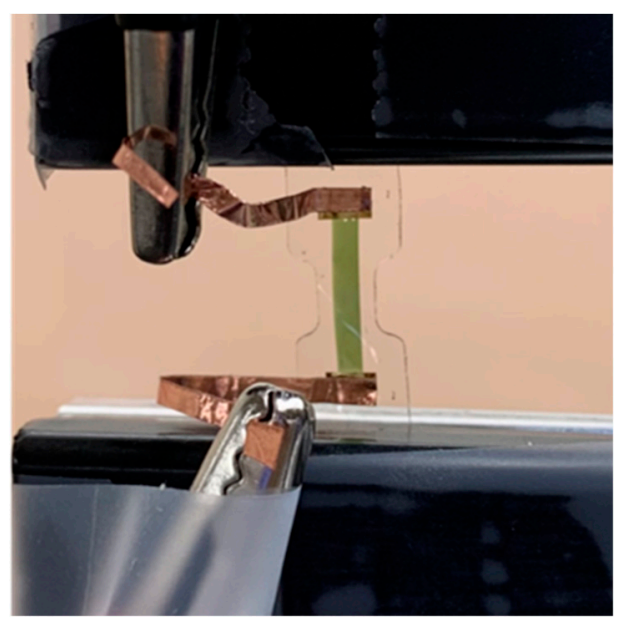

(b)

Figure 3. Measuring electrical properties. (a) Sample A (PANI strain sensor), (b) Sample B (PANI/Au strain sensor), and Sample C (PANI/Au/H-PDMS strain sensor).

\section{Results}

\subsection{PANI Morphology}

It was important to maintain the interconnection of PANI under high tension to have reversible tensile strength. Therefore, the construction of an island-bridge structure was significant because the previous study showed its effectiveness of up to $150 \%$ [9]. In the earlier study, we found that electrochemically synthesized PANI formed the initial thin film that served as an island, and the nanowires that grew on it acted as a bridge. Figure 4a shows the scanning electron microscope (SEM) image of PANI taken after transfer with PDMS, depicting island-bridge constitutions. Figure $4 \mathrm{~b}$ is the enlarged image, revealing the organization with small dots instead of nanowires. These small dots, the reverse side of the PANI film, appeared because the transfer process caused PDMS to embed it. Therefore, the nanowires of the island-bridge buried in this way were able to tolerate up to $50 \%$ strain.

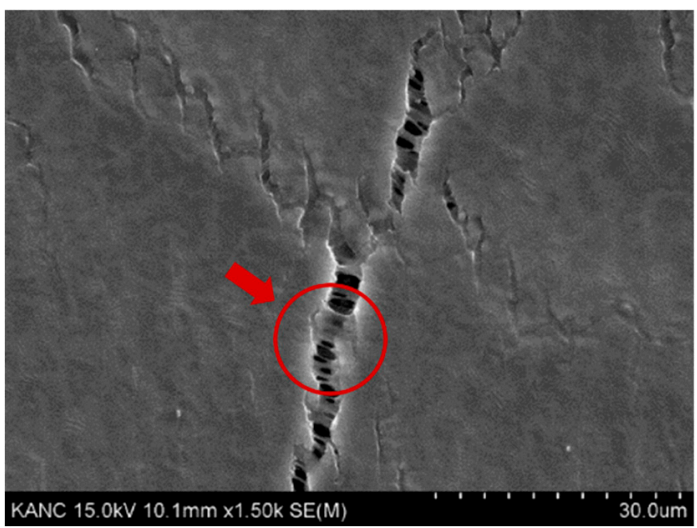

(a)

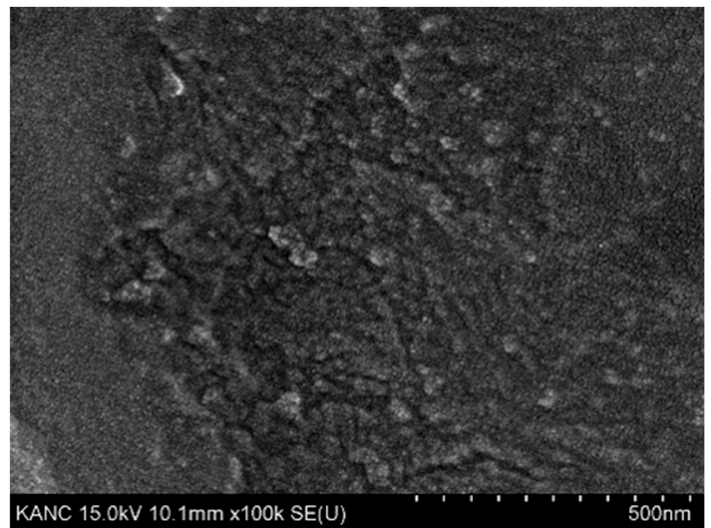

(b)

Figure 4. SEM image of transferred PANI film: (a) the island-bridge structure; (b) enlarged image of PANI film.

\subsection{The MCL Protection Effect of the Dumbbell Structure and the H-PDMS}

Figure 5 is an equivalent circuit model of the dumbbell shape of Sample C. Part 1 is the narrowest area, Part 2 is the curved area, and Part 3 is the widest area. The resistance of PANI formed in each area is denoted by $R_{1}, R_{2}, R_{3}$. The equation for the resistance of PANI $\left(R_{\text {pani }}\right)$ is expressed in Equation (1), and a Young's modulus of the S-PDMS $(E)$ is presented 
in Equation (2). $\rho$ is the resistivity of PANI, $l$ is the length of PANI in the corresponding part $\left(l_{1}=2.4 \mathrm{~mm}, l_{2}=1.6 \mathrm{~mm}, l_{3}=1.335 \mathrm{~mm}\right)$, and $A_{\text {pani }}$ is the cross-sectional area of PANI. Since $E$ is related to the S-PDMS, $F$ is the force applied to the S-PDMS, and $A_{p d m s}$ is the cross-sectional area of the S-PDMS $\left(A_{p d m s 1}=3 \mathrm{t} \mathrm{mm}, A_{p d m s 2} \approx 4.6 \mathrm{t} \mathrm{mm}, A_{p d m s 3}=6.26 \mathrm{t} \mathrm{mm}\right.$, $\mathrm{t}=$ thickness of the S-PDMS).

$$
\begin{gathered}
R_{\text {pani }}=\frac{\rho l}{A_{\text {pani }}} \\
E=\frac{\text { stress }}{\text { strain }}=\frac{F / A_{\text {pdms }}}{\Delta l / l}
\end{gathered}
$$
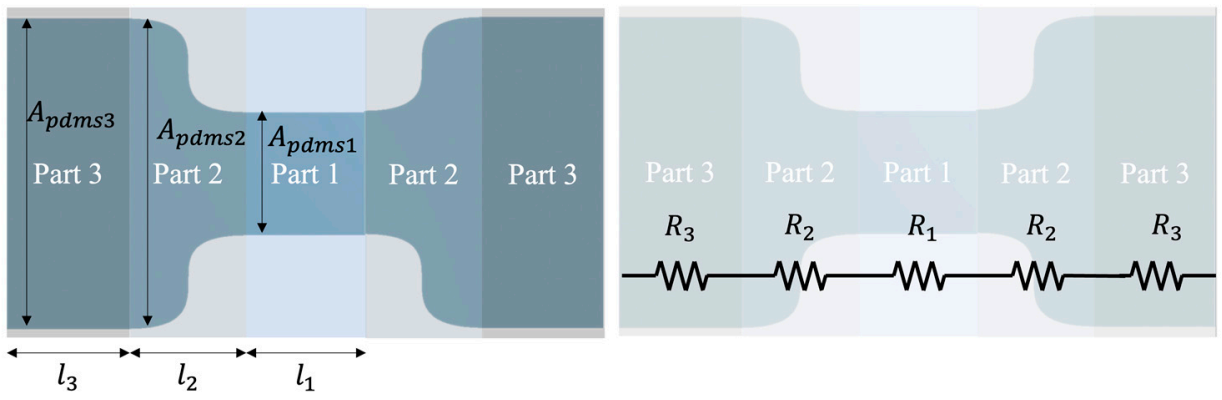

Figure 5. An equivalent circuit model of dumbbell shape.

The strain $(S)$ of Part 1 can be expressed as $S_{1}$ (Equation (3)). Then, the changes in $S$, when $A_{p d m s}$ are different, can be expressed in Equations (4) and (5).

$$
\begin{gathered}
\Delta l_{1} / l_{1}=S_{1} \\
S_{2}=S_{1} \frac{A_{p d m s 1}}{A_{p d m s 2}} \\
S_{3}=S_{1} \frac{A_{p d m s 1}}{A_{p d m s 3}}
\end{gathered}
$$

The change in resistance $\left(\Delta R_{\text {pani }}\right)$ can be obtained based on the change in PANI's length $(\Delta l)$ (Equations (6)-(8)). By referring to Equations (3)-(5), $\Delta l$ can be expressed by the equations related to $S_{1}$ (Equations (9) and (10)). As a result, the dumbbell structure is protective of the MCL because $\Delta l_{3}$ is the smallest.

$$
\begin{gathered}
\Delta R_{1}=\frac{\rho \Delta l_{1}}{A_{\text {pani }}} \\
\Delta R_{2}=\frac{\rho \Delta l_{2}}{A_{\text {pani }}} \\
\Delta R_{3}=\frac{\rho \Delta l_{3}}{A_{\text {pani }}} \\
\Delta l_{2}=l_{2} S_{2}=l_{2} S_{1} \frac{A_{1}}{A_{2}} \\
\Delta l_{3}=l_{3} S_{3}=l_{3} S_{1} \frac{A_{1}}{A_{3}}
\end{gathered}
$$

We compared the Ag/H-PDMS and Ag-only samples to verify the efficiency of the buffer layer and the dumbbell shape. We also observed the surface and the resistance according to strain. The strain-dependent surface changes of the Ag thin film in the $\mathrm{Ag} / \mathrm{H}-\mathrm{PDMS}$ and the Ag samples are shown in Figure $6 \mathrm{a}-\mathrm{f}$. At the initial state $(0 \%)$, the $\mathrm{Ag} / \mathrm{H}-\mathrm{PDMS}$ samples completely protected the surface (Figure 6a,c). However, the Ag 
samples immediately adhered to the S-PDMS and damaged the surface even in the absence of applying artificial stress (Figure 6b). In particular, the Ag/H-PDMS samples' initial condition was maintained even at $20 \%$ strain (Figure $6 \mathrm{~d}$ ). On the contrary, the Ag samples showed cracks both coincident with and perpendicular to the strain axis (Figure 6e).

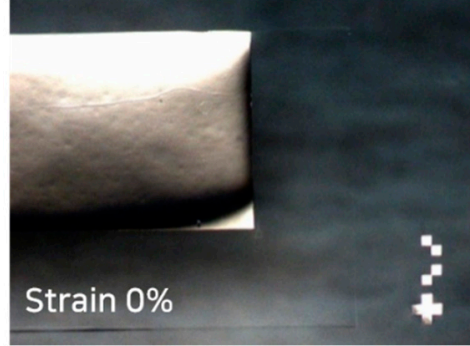

(a)

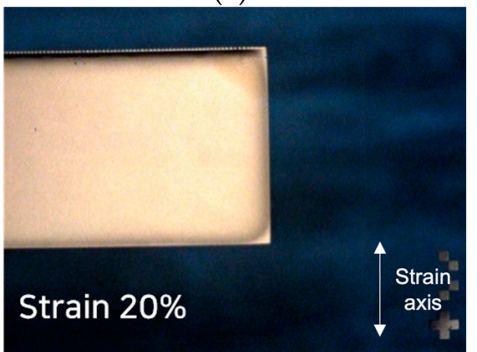

(d)

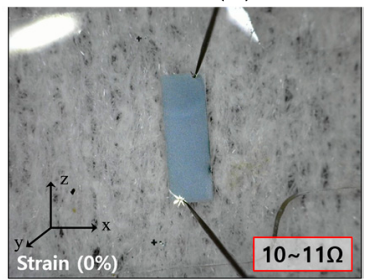

(g)

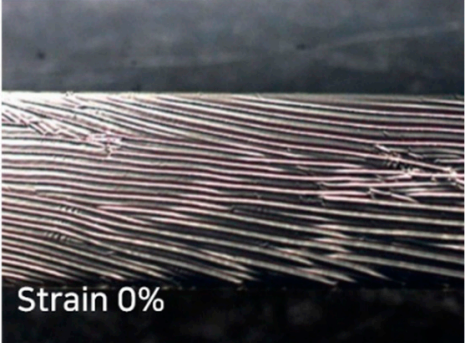

(b)

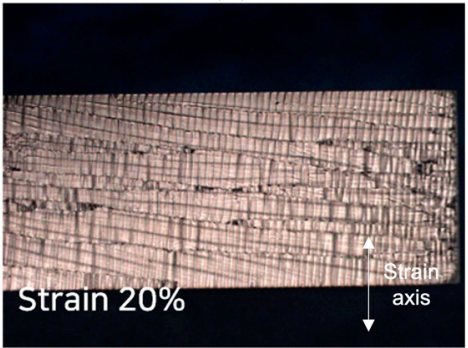

(e)

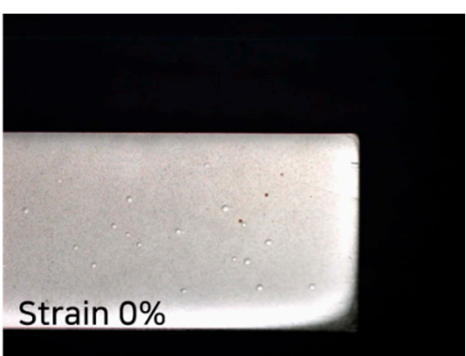

(c)

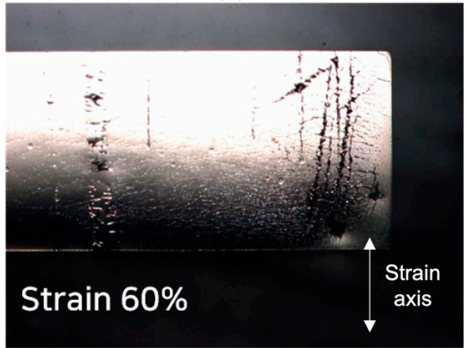

(f)

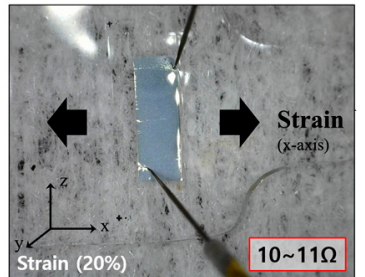

(h)

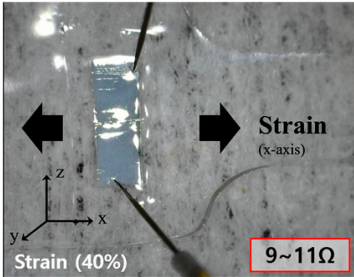

(i)

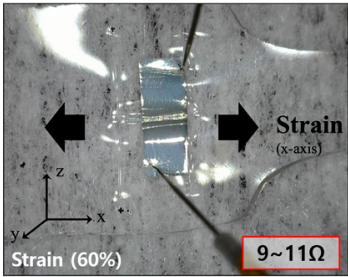

(j)

Figure 6. Ag/H-PDMS and Ag-only samples: (a) $0 \%$ of the $\mathrm{Ag} / \mathrm{H}-\mathrm{PDMS}$ sample; (b) $0 \%$ of the $\mathrm{Ag}$ sample; (c) $0 \%$ of the Ag/H-PDMS; (d) 20\% of the Ag/H-PDMS sample; (e) 20\% of the Ag sample; (f) $60 \%$ of the Ag/H-PDMS sample; (g-j) overall image of the Ag/H-PDMS sample and the measured resistance at (g) $0 \%$, (h) $20 \%$, (i) $40 \%$, and (j) $60 \%$.

Figure $6 \mathrm{~g}-\mathrm{j}$ shows the images of one side of the dumbbell shape, its Ag thin film, and the H-PDMS in the Ag/H-PDMS samples. Figure $6 \mathrm{~g}$, h shows that Ag thin film keeps its outline under deformation, under strain of up to $20 \%$. However, it also shows distortion in the $z$-axis at $40 \%$ and $60 \%$ strains, causing destruction in the same direction as the strain axis. As shown in Figure 6f, the Ag/H-PDMS samples experienced damage that was parallel to the strain axis under high tension.

Although the H-PDMS had sufficient rigidity to protect the Ag films from strain, the difference in elasticity between the S-PDMS and the H-PDMS, and their high flexibility, caused these cracks (Figure 6i,j). When stress was applied in the x-axis, the S-PDMS, having high elasticity, stretched in the same axis as the stress applied, and decreased in width in the $y$-axis at the same time. However, the S-PDMS beneath the H-PDMS could not be stretched due to the H-PDMS being at the top, with its low stretchability. Therefore, the H-PDMS-formed regions were bent in the $\mathrm{z}$-axis to compensate for the width. This implies that in the case of samples with the buffer layer, the parallel detriments appeared due to flexibility and not due to stretching.

The resistance measurement was carried out to evaluate the performance. The probes were positioned from edge to edge of the Ag thin film to maximize the effect of its breakage 
caused by deformation. Despite this damage, all the results measured at strains from $0 \%$ to $60 \%$, with a $20 \%$ interval, had $9-11 \Omega$, indicating stability.

\subsection{I-V Measurement}

We measured the I-V characteristics of Samples A, B, and C to evaluate the stability and elasticity of the MCL-adapted and the buffer layer-adapted strain sensor. Figure 7 shows the I-V measurements of each sample at strains of $0 \%, 10 \%, 20 \%, 30 \%, 40 \%$, and $50 \%$. Through the I-V curves, the linearity and reproducibility of each sample were exhibited. GF, a parameter indicating sensitivity, verified the ohmic contact of PANI and the MCL, and their stability. GF was calculated as the change in relative resistance $(\Delta R)$ with strain $(\varepsilon)$ (Equation (11)). The relative resistance change $(\Delta R)$ is described in Equation (12), where $R_{0}$ denotes the resistance in the initial state $(0 \%)$ and $R$ represents the resistance in the strain state. For the estimation, each resistance was described in the following colors: red for $0 \%$; orange for $10 \%$; yellow for $20 \%$; green for $30 \%$; blue for $40 \%$; and purple for $50 \%$. Figure 7 a shows an exponential aspect and randomly overlapped values of Sample A. At the 30\%, $40 \%$, and $50 \%$ strains, the currents were decreased, with increasing strain. However, the result was almost the same at $0 \%, 10 \%$, and $20 \%$, regardless of the strain. Instability was indicated with disparate results, despite repeated measurements under the same conditions. At $30 \%$, the first measurements had a value of approximately $0.015 \mathrm{~mA}$ (biased at $10 \mathrm{~V}$ ). However, the second measurement had a value of $0.005 \mathrm{~mA}$, showing an error of $67 \%$ even with the same estimation conditions. Additionally, the current at $10 \%$ strain was similar to that at $0 \%$ strain at a positive voltage bias, but the current was similar at $30 \%$ and $40 \%$ in the case of a negative voltage, rather than $0 \%$. This asymmetry implied that PANI in Sample A, with copper tape, had unstable contact. The I-V curves of Samples B and C showed more linear curves than did Sample A, as shown in Figure 7, because the MCL formed ohmic contact with PANI. However, Sample B was insulated at $40 \%$ strain and also emitted some noises. Figure 8 is the plot of resistance vs. strain when $5 \mathrm{~V}$ is applied. The resistance was measured consecutively three times at each strain. The elasticity of the strain sensor damaged the MCL, which caused noises under the strain during the measurements and had a fatal effect on strain determination. The linearity or tendency of resistance according to strain was not observed in Samples A and B (Figure 8a,b). In contrast, Sample C showed clear I-V curves and resistance according to each strain (Figures 7c and 8c). By employing the MCL and the H-PDMS, noises could be suppressed and could improve the sensor's reliability and stability.

$$
\begin{gathered}
\mathrm{GF}=\frac{\Delta R}{\varepsilon} \\
\Delta R=\frac{R-R_{0}}{R_{0}}
\end{gathered}
$$

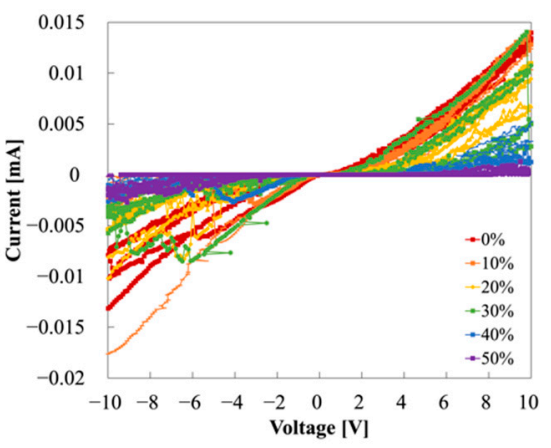

(a)

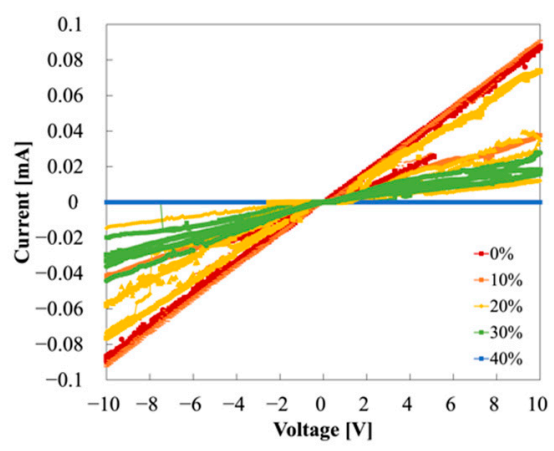

(b)

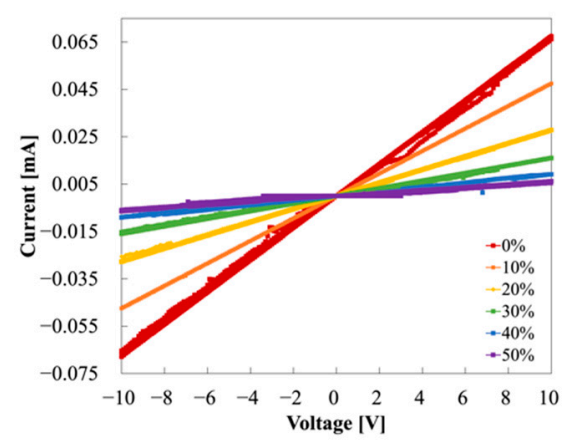

(c)

Figure 7. I-V measurement of Samples A-C: (a) Sample A (PANI strain sensor), (b) Sample B (PANI/Au strain sensor), and (c) Sample C (PANI/Au/H-PDMS strain sensor). 


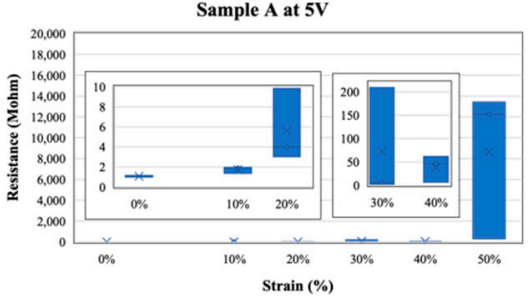

(a)

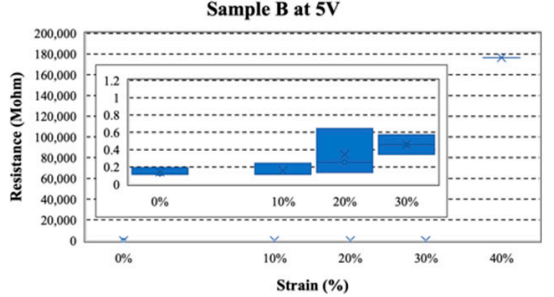

(b)

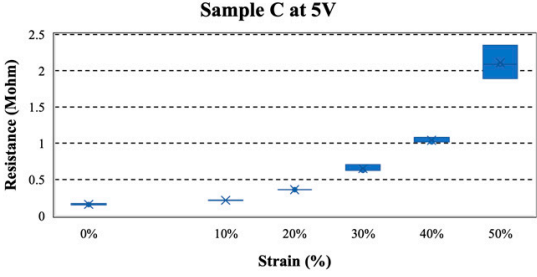

(c)

Figure 8. Resistance vs. strain of Sample A-C: (a) Sample A (Insets show magnified view due to large resistance differences), (b) Sample B (the inset shows a magnified view), and (c) Sample C showing distinguishable classification of the strain.

Figure 9 shows the individual graphs in each strain of Sample A. Figure $9 a-f$ show that Sample A has strain-dependent threshold voltages: $2 \mathrm{~V}$ at $0 \%$ and $10 \%$ strain; $2-4 \mathrm{~V}$ at $20 \%$ and $30 \%$ strain; and $4-6 \mathrm{~V}$ at $40 \%$ and $50 \%$ strain. These values indicate that the threshold voltage increases with increasing strain. Figure 9 also shows more sporadic data distribution and hysteresis with increasing strain. Hence, we observed that the current at $10 \mathrm{~V}$ of each strain generally decreased with rises in strain. However, currents at $20 \%$ strain had exceptional results, having larger values than $10 \%$. This irregularity can be interpreted according to the work function and contact resistance of copper tape and PANI, as mentioned above.

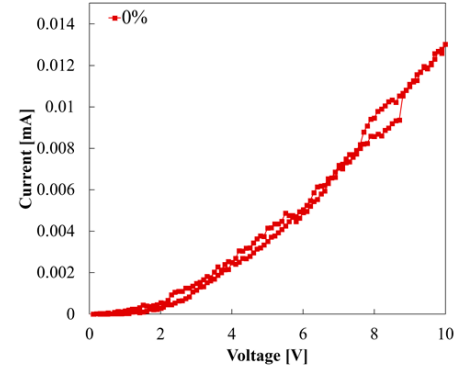

(a)

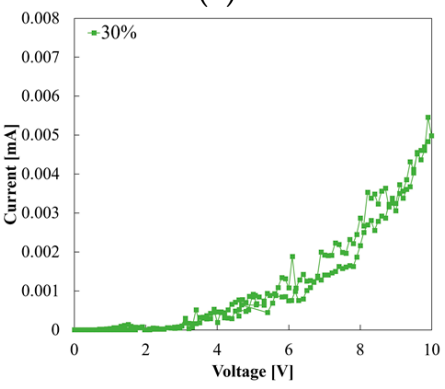

(d)

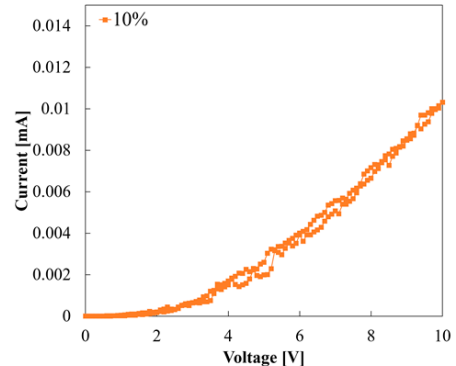

(b)

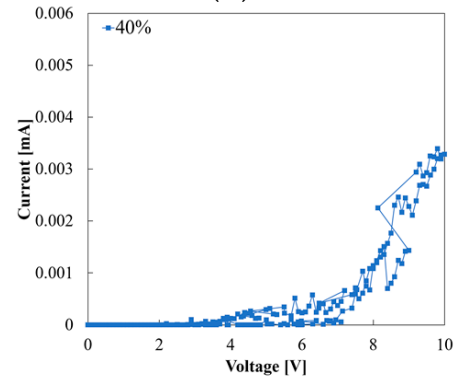

(e)

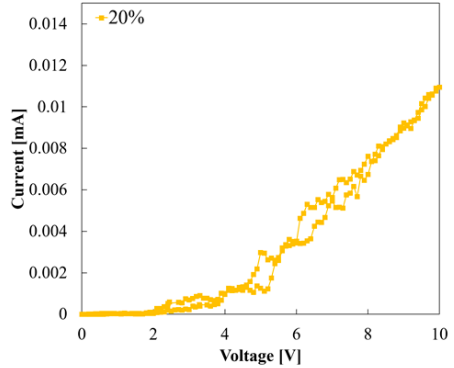

(c)

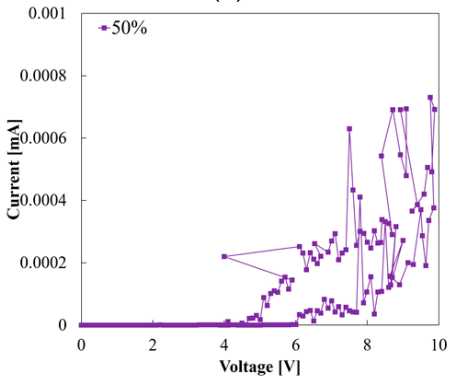

(f)

Figure 9. Individual I-V curves of Sample A based on strain: (a) $0 \%$ strain, (b) $10 \%$ strain, (c) $20 \%$ strain, (d) $30 \%$ strain, (e) $40 \%$ strain, and (f) $50 \%$ strain.

GFs of Sample A are shown in Figure 10. Considering GFs at 10\%, they were presented between 0 and 0.5 when positive voltage-biased, whereas they were around 0.5 at the negative voltages, showing an asymmetric distribution. Similarly, GFs were distributed between 0 and 0.5 at the positive voltages at 30\%; however, GFs were between 0.5 and 1 at the negative voltages. Notably, it was impossible to discriminate between the strain based on the GFs in these two cases because the GFs at the positive voltages were overlapped in the same range of $0-0.5$. At $20 \%$ and $40 \%$, GFs of both sides of the voltages showed a relatively symmetrical tendency. In contrast, GFs of 50\% had asymmetry, with GFs of 10 or more at the positive side and $0-5$ at the negative side. The unstable behavior in Sample A 
was illustrated through the GFs' distribution. The asymmetry of the GFs is the result of the Schottky junction of PANI and the copper tape.

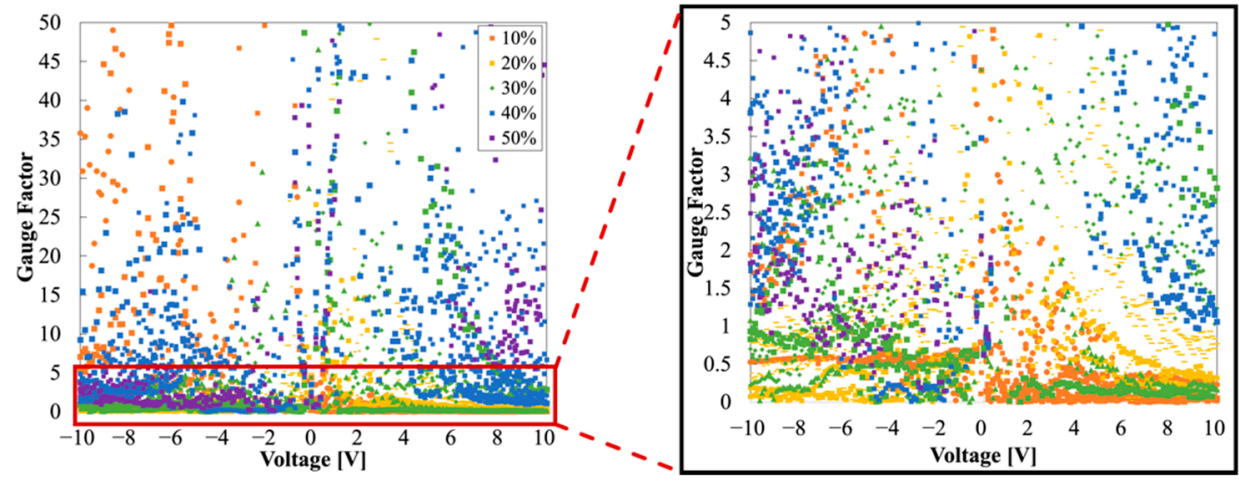

Figure 10. Overall GF of Sample A (left) and enlarged GF of Sample A (right).

The individual I-V graphs for each strain of Sample B are shown in Figure 11b-d. Each graph is the result of three consecutive measurements under the same conditions. Sample B used Au as the MCL, which has a larger work function than PANI, and a stable ohmic contact can be formed. Thus, Sample B was significantly more linear than Sample A at $0 \%$ strain. However, Sample B showed undesirable behaviors, such as large fluctuations in the I-V curves through multiple measurements when the strain was applied as shown in Figure 11b,c. The I-V curves for $10 \%$ strain shown in Figure $11 \mathrm{~b}$ indicate that the first measurement has the lowest value, and the second and third measurements have increased. The MCL was damaged as the strain applied, and the resistance rose rapidly and exhibited its lowest value in the first trial. It partially recovered and stabilized due to the elasticity of the S-PDMS. The resistance caused the elevated currents in the second and third trials. Similarly, the graph for $20 \%$ in Figure 11c depicts that the first trial had the lowest value and current increased in the second trial and decreased in the third. As with Figure 11b, Figure 11c shows a drastic and immediate rise in the resistance at the moment when stress was applied. The damaged MCL recovered with time, leading to the current increase at the second measurement. The third measurement was lower than the second one in contrast to the interpretation described at $10 \%$ strain. This might be explained by the fact that the MCL was damaged when the strain was applied, even though the dumbbell structure and the elasticity of the S-PDMS protected the MCL. Therefore, the resistance increased, negatively affecting the sensor's behavior under the strain. At 30\%, the I-V curves had the lowest value at the first trial, increasing at the second and third trials (Figure 11d). The range of current fluctuations according to the number of trials was small, unlike the previous range at $10 \%$ and $20 \%$, which was different from the other two cases where the damage to the MCL was noticeably recovered by the stretchability of the substrate. At $30 \%$, the MCL could not revive its properties, or it may have needed sufficient time to recuperate because of the large stress and deformation. Therefore, the MCL with the H-PDMS would be truly necessary.

The GFs of Sample B are shown in Figure 12. GFs at $40 \%$ were over 1,000,000 because of the MCL's destruction. GFs at 10\%, 20\%, and 30\% were spread between 0 and 10 and made it difficult to discriminate among the strains, owing to their wide distributions. Additionally, fluctuation in hysteresis and the unsettled results following the damaged MCL negatively affected the sensor performance. 


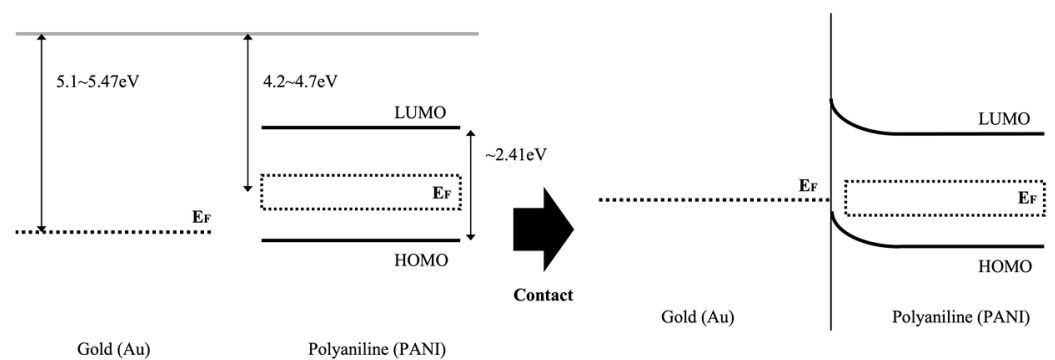

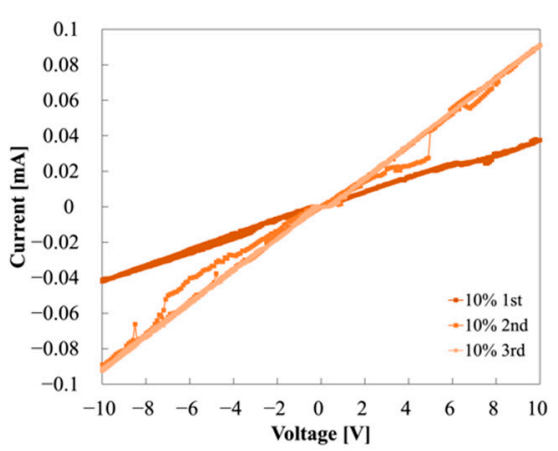

(b) (a)

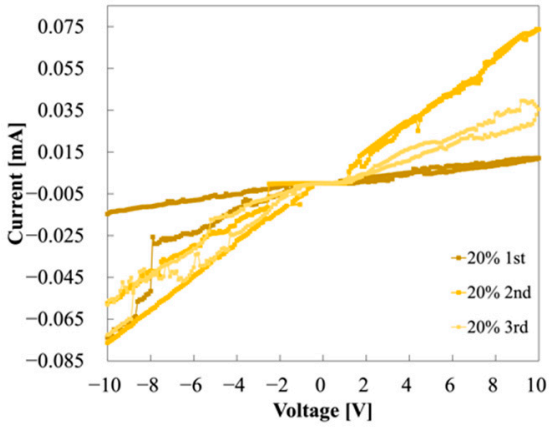

(c)

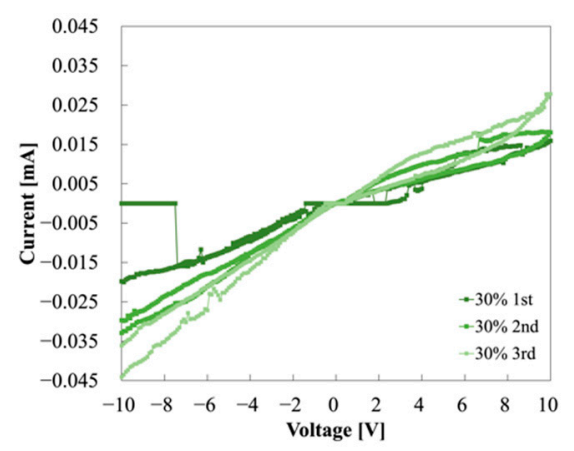

(d)

Figure 11. (a) Energy band diagram of PANI and the MCL before contact (left) and after contact (right); the individual I-V curves according to strain in Sample B: (b) 10\% of strain, (c) 20\% of strain, and (d) $30 \%$ of strain.

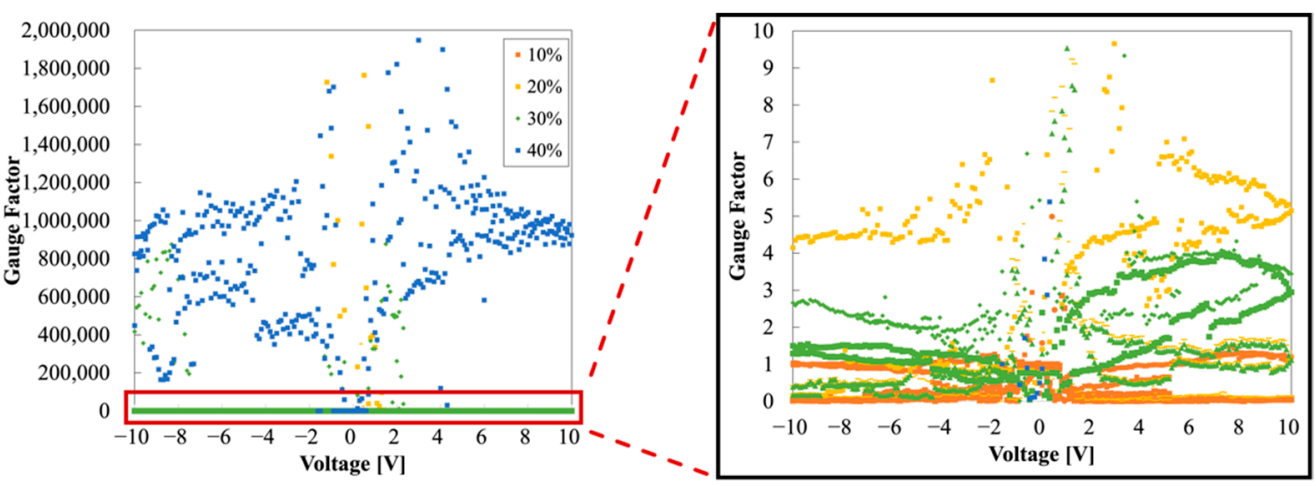

Figure 12. Overall GF of Sample B (left) and enlarged GF of Sample B (right).

As shown in Figure 7c, Sample $\mathrm{C}$ has clearly differentiated I-V measurements for each strain, as the most linear, stable, and repeatable. Specifically, the strain was linear and distinguishable even at the low voltages of $-2 \mathrm{~V}-2 \mathrm{~V}$, as shown in Figure 13a. Furthermore, when comparing the currents at $2 \mathrm{~V}$ in the initial state of Samples A and C, the current in the latter was 48 times larger than in the former. The MCL and PANI connection had smaller contact resistance than the copper tape and PANI connection. The GFs of Sample C are shown in Figure 13b: GFs of 0.5 at $10 \%$, GFs of 1 at $20 \%$, GFs of 2.5 at $30 \%$, GFs of $4-5$ at $40 \%$, and GFs of more than 6 at $50 \%$. The GFs were completely differentiated and characterized according to each strain. Moreover, the ohmic contact between PANI and the MCL was verified via the symmetric GFs distribution through the positive and negative sides. In general, a high GF indicates high sensitivity of the strain sensor, but at the same time, high-precision and expensive equipment is required to detect large resistance $\left(>10^{9} \Omega\right)$ [7]. Table 1 shows the gauge factors, depending on the measurement contact method. Therefore, Sample C, with a relatively small but highly distinguishable GF will be promising for further body-mounted wearable device applications. 


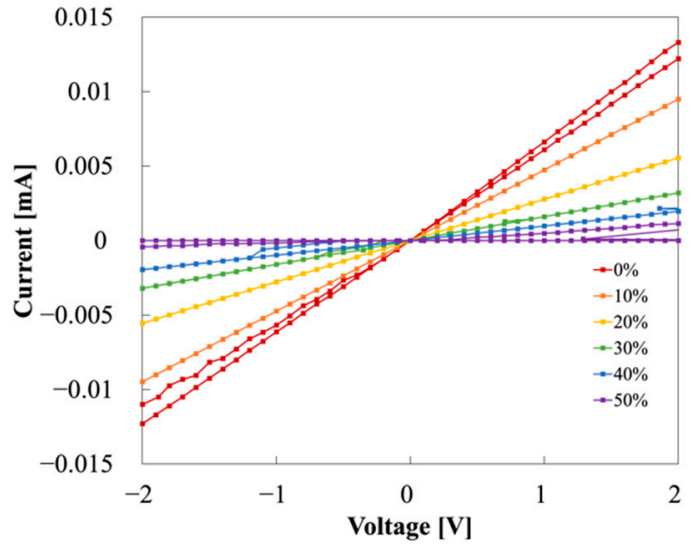

(a)

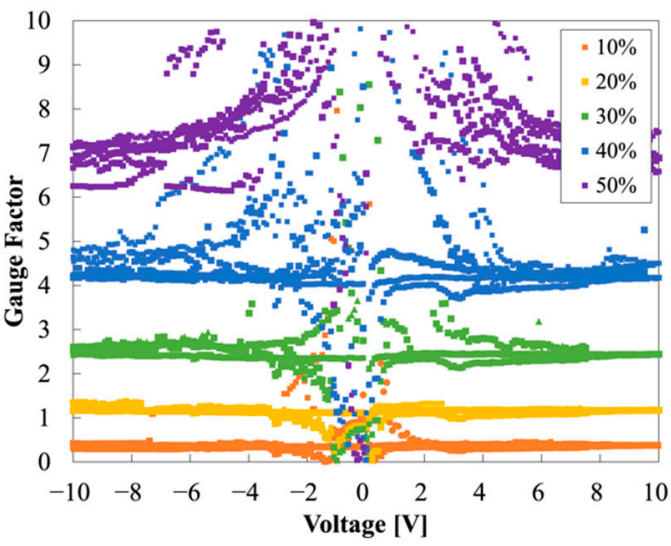

(b)

Figure 13. (a) I-V characteristics of Sample $C$ with a voltage range $-2 \mathrm{~V}-2 \mathrm{~V}$; (b) GFs of Sample C.

Table 1. The comparison of the measurement contact method of PANI-based strain sensors.

\begin{tabular}{cccc}
\hline $\begin{array}{c}\text { Measurement Contact } \\
\text { Method }\end{array}$ & Gauge Factor & Materials & Ref. \\
\hline Copper tape and silver glue & 54 at $50 \%$ strain & PANI & {$[7]$} \\
Copper tape and silver paste & 140 at $100 \%$ strain & PANI & {$[9]$} \\
Silver fabric & 74 at $1 \%$ strain & PANI & {$[31]$} \\
Copper wire and silver paste & 6.725 at $0 \sim 120 \%$ strain & PANI/TPU & {$[32]$} \\
MCL & 6 at $50 \%$ & PANI & This work \\
\hline
\end{tabular}

Figure 14 shows the I-V curves of PANI/Au/H-PDMS strain sensors with three different PANI lengths. Each inset shows a sensor with different PANI lengths: $5020 \mu \mathrm{m}$, $7020 \mu \mathrm{m}$, and $8270 \mu \mathrm{m}$, respectively. The I-V measurement of the strain sensor with the PANI length of $5020 \mu \mathrm{m}$ is shown in Figure 14a. We observed the insulation due to the rapid rise of resistance under stress with increasing strain from $20 \%$ to $30 \%$. When the length of PANI shortened, the MCL and H-PDMS were positioned on the curved area of the dumbbell, no longer protected by the structure. The I-V measurements of the strain sensors with PANI lengths of $7020 \mu \mathrm{m}$ and $8270 \mu \mathrm{m}$ are shown in Figure $14 \mathrm{~b}, \mathrm{c}$, respectively. They had distinctly isolated values according to deformation and sustained conductivity, even at 50\% strain. Both samples, the MCL and the H-PDMS, placed on a wide area of the dumbbell, were structurally sufficient to protect it from the strain, resulting in stable outcomes.

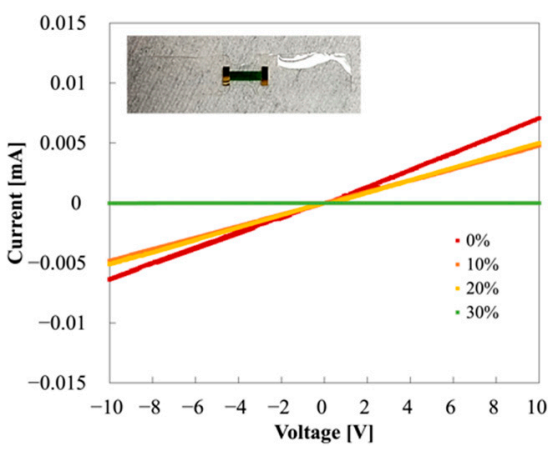

(a)

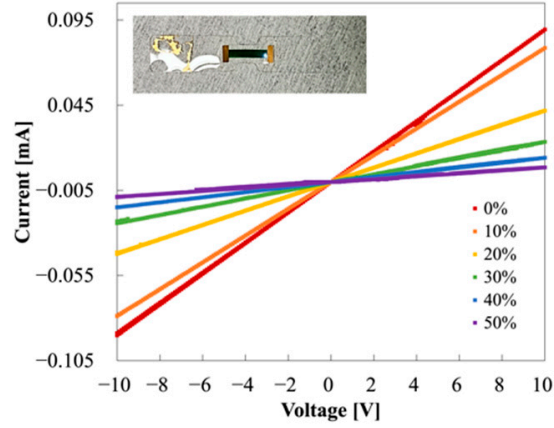

(b)

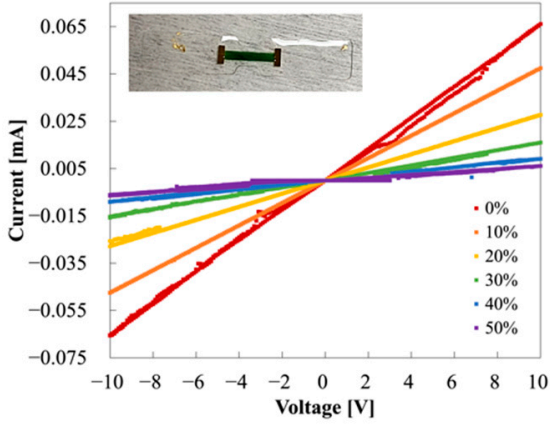

(c)

Figure 14. I-V curves of the PANI/Au/H-PDMS strain sensor according to the different lengths of PANI: (a) $5020 \mu \mathrm{m}$, (b) $7020 \mu \mathrm{m}$, and (c) $8270 \mu \mathrm{m}$. 


\subsection{Transient Measurement}

Figure 15a shows the transient measurement of Sample C, which was pulled and recovered at the speed of $50 \mathrm{~mm} / \mathrm{min}$ and $10 \mathrm{~s}$ of waiting time. It shows the relative resistance changes with time, at 10\%, 20\%, and 30\% strain. The relative resistance change at $20 \%$ strain is two times greater than that of $10 \%$, and that at $30 \%$ is four times greater than that of $20 \%$. This indicates that the relative resistance change can be a parameter for classifying deformation. In particular, intense overshooting occurred with increasing strain due to the rapid and instantaneous changes in the relative resistance caused by the sudden increase in resistance at the moment of pulling. Figure 15b, which was pulled and recovered at the speed of $6 \mathrm{~mm} / \mathrm{min}$, indicates a gradual relative resistance change without the overshooting phenomenon. Consequently, it demonstrates that relative resistance change can be a parameter for classifying deformation but can be affected by tensile speed.

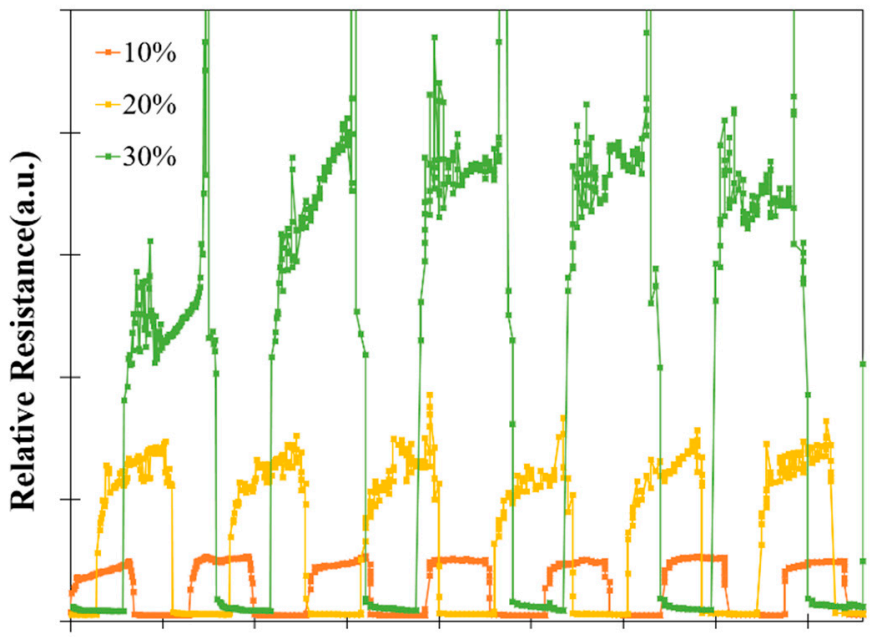

Time [s]

(a)

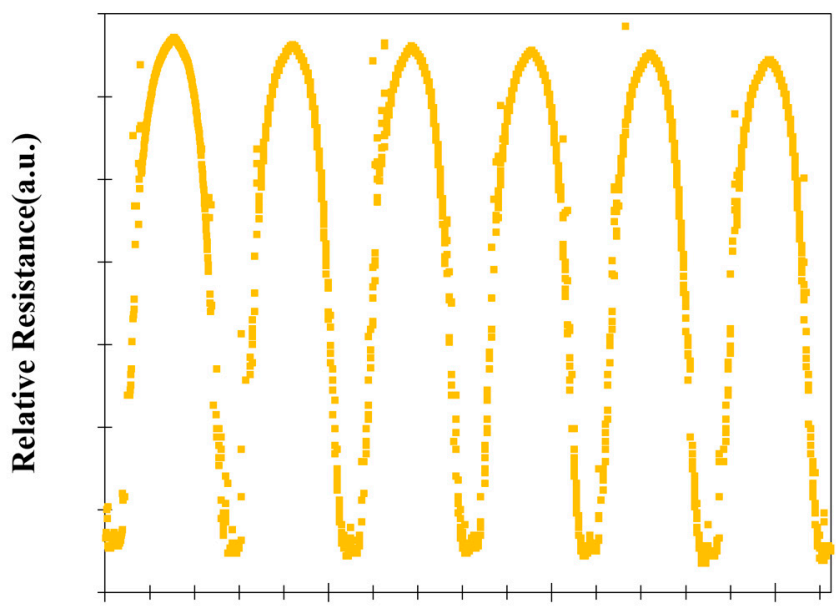

Time [s]

(b)

Figure 15. Transient measurement of Sample C: (a) relative resistance change as a function of time at $10 \%, 20 \%$, and $30 \%$ strain; (b) transient curve at the tensile speed of $6 \mathrm{~mm} / \mathrm{min}$ and a waiting time of $0.1 \mathrm{~s}$.

\section{Conclusions}

We introduced the strain sensor to the adapting MCL to ensure ohmic contact with PANI and formed a buffer layer to protect it from the strain. The $\mathrm{I}-\mathrm{V}$ curves according to each strain showed that the sample containing only PANI had the most unstable behavior and suggested that PANI and copper tape had Schottky contact, prolonging performance degradation. The strain sensor with the MCL, but without the H-PDMS, had deterioration due to damage to the MCL under deformation. The PANI/Au/H-PDMS strain sensor had stable behavior that helped in distinguishing the characterization and classification. As a result, we demonstrated the improvement of operational stability by adapting the MCL and the buffer layer. Moreover, this arrangement is more suitable for body-mounted wearable devices because it is stable even at a low voltage. The fabrication of the substrate with different stretchability regions on a single plane provided a research foundation for strain sensors' integration. We anticipate various future research into structural enhancement, such as the measurement or packaging of a strain sensor, rather than the development of materials, which can improve a device's performance and sustainability, to contribute to the commercialization of stretchable devices.

Author Contributions: Methodology, conceptualization, formal analysis, and writing - original draft, J.-Y.C. and E.-B.J.; formal analysis and data curation, H.-K.Y.; software, J.-H.H.; methodology and visualization, C.-J.Y.; software and validation, J.-H.L.; resources, funding acquisition, supervision, 
and writing - review and editing, H.-S.K. All authors have read and agreed to the published version of the manuscript.

Funding: This work was supported by the Mid-career Researcher Program through the National Research Foundation of Korea (NRF), funded by the Ministry of Science and ICT (No. 2019R1A2C2086747) and the MOTIE (Ministry of Trade, Industry, and Energy) in Korea, under the Fostering Global Talents for Innovative Growth Program supervised by the Korea Institute for Advancement of Technology (No. P0017307).

\section{Institutional Review Board Statement: Not applicable.}

Conflicts of Interest: The authors declare no conflict of interest.

\section{References}

1. Tan, C.; Dong, Z.; Li, Y.; Zhao, H.; Huang, X.; Zhou, Z.; Jiang, J.W.; Long, Y.Z.; Jiang, P.; Zhang, T.Y.; et al. A high performance wearable strain sensor with advanced thermal management for motion monitoring. Nat. Commun. 2020, 11, 3530. [CrossRef] [PubMed]

2. Amjadi, M.; Pichitpajongkit, A.; Lee, S.; Ryu, S.; Park, I. Highly stretchable and sensitive strain sensor based on silver nanowireelastomer nanocomposite. ACS Nano 2014, 8, 5154-5163. [CrossRef]

3. Horev, Y.D.; Maity, A.; Zheng, Y.; Milyutin, Y.; Khatib, M.; Yuan, M.; Suckeveriene, R.Y.; Tang, N.; Wu, W.; Haick, H. Stretchable and Highly Permeable Nanofibrous Sensors for Detecting Complex Human Body Motion. Adv. Mater. 2021, 33, 2102488. [CrossRef] [PubMed]

4. Yu, G.F.; Yan, X.; Yu, M.; Jia, M.Y.; Pan, W.; He, X.X.; Han, W.P.; Zhang, Z.M.; Yu, L.M.; Long, Y.Z. Patterned, highly stretchable and conductive nanofibrous PANI/PVDF strain sensors based on electrospinning and in situ polymerization. Nanoscale 2016, 8, 2944-2950. [CrossRef]

5. Chen, J.; Zhu, Y.; Jiang, W. A stretchable and transparent strain sensor based on sandwich-like PDMS/CNTs/PDMS composite containing an ultrathin conductive CNT layer. Compos. Sci. Technol. 2020, 186, 107938. [CrossRef]

6. Chen, S.; Wei, Y.; Yuan, X.; Lin, Y.; Liu, L. A highly stretchable strain sensor based on a graphene/silver nanoparticle synergic conductive network and a sandwich structure. J. Mater. Chem. C 2016, 4, 4304-4311. [CrossRef]

7. Gong, X.X.; Fei, G.T.; Fu, W.B.; Fang, M.; Gao, X.D.; Zhong, B.N.; Zhang, L.D. Flexible strain sensor with high performance based on PANI/PDMS films. Org. Electron. 2017, 47, 51-56. [CrossRef]

8. Song, E.; Choi, J.W. Conducting polyaniline nanowire and its applications in chemiresistive sensing. Nanomaterials 2013, 3 , 498-523. [CrossRef]

9. Cai, G.; Wang, J.; Lin, M.F.; Chen, J.; Cui, M.; Qian, K.; Li, S.; Cui, P.; Lee, P.S. A semitransparent snake-like tactile and olfactory bionic sensor with reversibly stretchable properties. NPG Asia Mater. 2017, 9, e437. [CrossRef]

10. Amjadi, M.; Kyung, K.U.; Park, I.; Sitti, M. Stretchable, skin-mountable, and wearable strain sensors and their potential applications: A review. Adv. Funct. Mater. 2016, 26, 1678-1698. [CrossRef]

11. Tolvanen, J.; Hannu, J.; Jantunen, H. Stretchable and washable strain sensor based on cracking structure for human motion monitoring. Sci. Rep. 2018, 8, 13241. [CrossRef] [PubMed]

12. Abdulrazzaq, O.; Bourdo, S.E.; Saini, V.; Watanabe, F.; Barnes, B.; Ghosh, A.; Biris, A.S. Tuning the work function of polyaniline via camphorsulfonic acid: An X-ray photoelectron spectroscopy investigation. RSC Adv. 2015, 5, 33-40. [CrossRef]

13. Hajibadali, A.; Nejhad, M.B.; Farzi, G.; Rad, H.H. Fabrication and characterization of polyaniline based Schottky diode. In Proceedings of the 2013 21st Iranian Conference on Electrical Engineering (ICEE), Mashhad, Iran, 14-16 May 2013.

14. Ghorbani, L.; Nasirian, S. Zinc oxide nanorods assisted by polyaniline network as a flexible self-powered Ultraviolet photodetector: A comprehensive study. Appl. Surf. Sci. 2020, 527, 146786. [CrossRef]

15. Li, Q.; Li, J.; Tran, D.; Luo, C.; Gao, Y.; Yu, C.; Xuan, F. Engineering of carbon nanotube/polydimethylsiloxane nanocomposites with enhanced sensitivity for wearable motion sensor. J. Mater. Chem. C 2017, 5, 11092-11099. [CrossRef]

16. Huang, L.; Wang, H.; Wu, P.; Haung, W.; Gau, W.; Fang, F.; Cai, N.; Chen, R.; Zhu, Z. Wearable flexible strain sensor based on three-dimensional wavy laser-induced graphene and silicone rubber. Sensors 2020, 20, 4266. [CrossRef] [PubMed]

17. Zheng, Y.; Li, Y.; Dai, K.; Wang, Y.; Zheng, G.; Liu, C.; Shen, C. A highly stretchable and stable train sensor based on hybrid carbon nanofillers/polydimethylsiloxane conductive composites for large human motions monitoring. Compos. Sci. Technol. 2018, 156, 276-286. [CrossRef]

18. Mata, A.; Fleischman, A.J.; Roy, S. Characterization of polydimethylsiloxane (PDMS) properties for biomedical micro/nanosystems. Biomed. Microdevices 2005, 7, 281-293. [CrossRef] [PubMed]

19. Johnston, I.D.; McCluskey, D.K.; Tan, C.K.L.; Tracey, M.C. Mechanical characterization of bulk Sylgard 184 for microfluidics and microengineering. J. Micromech. Microeng. 2014, 24, 035017. [CrossRef]

20. Jin, C.; Ma, C.; Yang, Z.; Lin, H. A force measurement method based on flexible PDMS grating. Appl. Sci. 2020, 10, 2296. [CrossRef]

21. Chen, W.; Lam, R.H.W.; Fu, J. Photolithographic surface micromachining of polydimethylsiloxane (PDMS). Lab Chip 2012, 12, 391-395. [CrossRef] 
22. Guo, L.; Deweerth, S.P. An Effective Lift-Off Method for Patterning High-Density Gold Interconnects on an Elastomeric Substrate. Small 2010, 6, 2847-2852. [CrossRef]

23. Van Dam, R.M. Solvent-Resistant Elastomeric Microfluidic Devices and Applications; California Institute of Technology: Pasadena, CA, USA, 2006.

24. ASTM D412-16; Standard Test Methods for Vulcanized Rubber and Thermoplastic Elastomer. ASTM: West Conshohocken, PA, USA, 2009; 1-14.

25. Mazurek, P.; Hvilsted, S.; Skov, A.L. Green silicone elastomer obtained from a counterintuitively stable mixture of glycerol and PDMS. Polymer 2016, 86, 1-7. [CrossRef]

26. Jeronimo, K.; Koutsos, V.; Cheung, R.; Mastropaolo, E. PDMS-ZnO Piezoelectric Nanocomposites for pressure sensors. Sensors 2021, 17, 5873. [CrossRef] [PubMed]

27. Sunkara, V.; Park, D.K.; Cho, Y.K. Versatile method for bonding hard and soft materials. RSC Adv. 2012, 2, 9066-9070. [CrossRef]

28. Dumond, J.J.; Low, H.Y.; Lee, H.P.; Fuh, J.Y. Multi-functional silicone stamps for reactive release agent transfer in UV roll-to-roll nanoimprinting. Mater. Horiz. 2016, 3, 152-160. [CrossRef]

29. Schmid, H.; Michel, B. Siloxane polymers for high-resolution, high-accuracy soft lithography. Macromolecules 2000, 33, 3042-3049. [CrossRef]

30. Hill, S.; Qian, W.; Chen, W.; Fu, J. Surface micromachining of polydimethylsiloxane for microfluidics applications. Biomicrofluidics 2016, 10, 054114. [CrossRef] [PubMed]

31. Kang, S.; Rachim, V.P.; Baek, J.H.; Lee, S.Y.; Park, S.M. A flexible patch-type strain sensor based on polyaniline for continuous monitoring of pulse wave. IEEE Access 2020, 8, 152105-152115. [CrossRef]

32. Tong, L.; Wang, X.X.; He, X.X.; Nie, G.D.; Zhang, J.; Zhang, B.; Guo, W.Z.; Long, Y.Z. Electrically conductive TPU nanofibrous composite with high stretchable for flexible strain sensor. Nanoscale Res. Lett. 2018, 13, 86. [CrossRef] [PubMed] 